Z Rheumatol 2019.78:116-118

https://doi.org/10.1007/s00393-018-0575-6

(C) Springer Medizin Verlag GmbH, ein Teil von Springer Nature 2018

CrossMark

\section{U. Lange ${ }^{1} \cdot$ W. $\mathrm{Mau}^{2}$}

${ }^{1}$ Abteilung Rheumatologie und klinische Immunologie, Kerkhoff-Klinik, Justus-Liebig-Universität Gießen, Bad Nauheim, Deutschland

${ }^{2}$ Institut für Rehabilitationsmedizin, Medizinische Fakultät, Martin-Luther-Universität Halle-Wittenberg, Halle (Saale), Deutschland

\title{
Nichtmedikamentöse Behandlung und Rehabilitation
}

Nach den Daten der Kerndokumentation aus dem Jahr 2014 erreichen von über 8000 Patienten mit rheumatoider Arthritis (RA) unter der medikamentösen Standardtherapie (Glukokortikoide, „disease modifying antirheumatic drugs" [DMARDs] und Biologika) nur $36 \%$ eine Remission. Somit ist bei einem Großteil der Patienten mit vermeidbaren Schmerzen, Funktionseinschränkungen, Arbeitsausfall und einer umfassenden Einschränkung der Lebensqualität zu rechnen. Maßnahmen der physikalischen Medizin als auch Verfahren der Rehabilitation nehmen somit eine zentrale Rolle bei der rheumatologischen Versorgung ein, insbesondere wenn es darum geht, Einschränkungen in Aktivitäten und Teilhabe $\mathrm{zu}$ reduzieren oder zu kompensieren, um eine adäquate Alltags- und Lebensführung zu erreichen.

\section{》) Physikalische Medizin und Rehabilitation spielen eine zentrale Rolle bei der rheumatologischen Versorgung}

Während in Deutschland auch international wegweisende Informationen zur Versorgung durch Rheumatologen vorliegen, die sich an der Kerndokumentation beteiligen, wissen wir wenig über die ambulante Behandlung von an RA Erkrankten in der Bevölkerung. Diese Lücke schließt die PROCLAIRStudie von Versicherten einer großen bundesweiten Krankenkasse, die Routinedaten mit Angaben der Patienten zusammenführt. Jacobs et al. zeigen, dass im Jahr 2015 weniger als die Hälfte der an RA Erkrankten eine Verordnung für physikalische Therapie erhielt, v. a. Krankengymnastik (35\%) bzw. manuelle Therapie (15\%), sehr selten Ergotherapie (3\%). Bei deutlicher Einschränkung der Alltagsfunktion (FFbH $<50)$ wird physikalische Therapie zwar häufiger verordnet als bei eher guter Funktion (Funktionsfragebogen Hannover $[\mathrm{FFbH}]>70), 4$ von 10 dieser stark eingeschränkten Patienten erhielten aber keine physikalische Therapie! Obwohl Beschränkungen der Heilmittelverordnung für RA entfallen sind und auch die aktuelle vierte Revision der interdisziplinären Leitlinie zum Management der frühen RA die rheumatologisch koordinierte multimodale Versorgung betont, stellten Rheumatologen diese Rezepte deutlich seltener aus (16\%) als Hausärzte $(42 \%)$ oder Orthopäden/Chirurgen (26\%).

Obwohl die große Bedeutung der Bewegungsaktivität und -therapie für die Funktionsfähigkeit von Patienten mit rheumatischen Erkrankungen vielfältig belegt ist, stellt die konsequente Durchführung in der Versorgung und im Alltag der Betroffenen eine beträchtliche Herausforderung dar. Das gilt noch mehr für ältere Kranke, die verstärkt durch Kound Multimorbidität sowie geriatrische Symptome eingeschränkt sind. Ausgehend von den im Jahr 2016 veröffentlichten „Nationalen Empfehlungen für Bewegung und Bewegungsförderung “ geben Golla et al. konkrete praxisnahe Hinweise für eine individuell adaptierte Umsetzung dieser Empfehlungen in der rheumatologischen Versorgung und im Alltag von älteren Patienten mit rheumatischen Erkrankungen.

Die multimodale rheumatologische Komplexbehandlung (MRKB) stellt ein Versorgungsinstrument für Patienten mit rheumatischen Erkrankungen dar, die aufgrund der Krankheitsschwere, der Akuität und der Funktionseinschränkungen eine akut-stationäre Behandlung benötigen. Dabei kommen im Rahmen der multimodalen schmerztherapeutischen und funktionsorientierten Behandlung ( $11 \mathrm{~h} /$ Woche) physikalische Therapiemaßnahmen in hoher Intensität zum Einsatz. Klemm et al. präsentieren eine monozentrische Retrospektivanalyse mit hochinteressanten und spannenden Daten zu den Wirkeffekten der MRKB.

Natürliche Heilmittel wie der Heiltorf haben ihren festen Stellenwert im differenzialindikativen physikalisch-medizinischen Behandlungskonzept, insbesondere in der Rehabilitationsmedizin. Eine Wirkung resultiert über 2 Wege: einerseits mechanische, thermische und chemische Soforteffekte, andererseits führt die serielle Applikation als Reizserientherapie zu lang anhaltenden physiologischen Umstellungen.

Dischereit et al. fokussieren in ihrem Beitrag auf die Wirkeffekte der seriellen Heiltorftherapie bei entzündlich rheumatischen wie auch degenerativen Erkrankungen. Insbesondere die Einflüsse auf die inflammatorischen Prozesse und das Zytokinmilieu untermauern im Zeitalter der evidenzbasierten Medizin eindeutig Wirksamkeitsnachweise auf mole- 
Hier steht eine Anzeige.

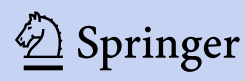


kularer Ebene und geben neue Einblicke in ein altbewährtes Heilmittel.

Mögen den Leserinnen und Lesern die vorliegenden Beiträge wichtige und interessante Einblicke vermitteln.

Ihre

\section{le. lount}

Univ.-Prof. Dr. med. Uwe Lange

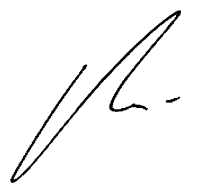

Univ.-Prof. Dr. med. Wilfried Mau

\section{Korrespondenzadresse}

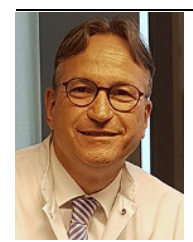

Univ.-Prof. Dr. U. Lange

Abteilung Rheumatologie und klinische Immunologie,

Kerkhoff-Klinik, Justus-LiebigUniversität Gießen Benekestr. 2-8, $61231 \mathrm{Bad}$ Nauheim, Deutschland u.lange@kerckhoff-klinik.de

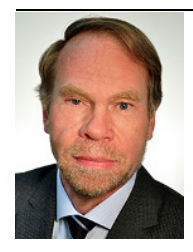

Univ.-Prof. Dr. W. Mau Institut für Rehabilitationsmedizin, Medizinische Fakultät, Martin-Luther-

Universität Halle-Wittenberg

Magdeburger Str. 8, 06097 Halle (Saale),

Deutschland wilfried.mau@

medizin.uni-halle.de

Interessenkonflikt. U. Lange und W. Mau geben an, dass kein Interessenkonflikt besteht.

Hier steht eine Anzeige.

\section{Springer}

